\title{
Evaluation of the Cost of Intelligent Upgrades of Transportation Infrastructure for Intelligent Connected Vehicles
}

\author{
Zongwei Liu,, ${ }^{1,2}$ Haokun Song, ${ }^{1,2}$ Hong Tan, ${ }^{1,2}$ Han Hao, ${ }^{1,2}$ and Fuquan Zhao $\mathbb{D}^{1,2}$ \\ ${ }^{1}$ State Key Laboratory of Automobile Safety and Energy, Tsinghua University, Beijing 100084, China \\ ${ }^{2}$ Tsinghua Automobile Strategy Research Institute, Tsinghua University, Beijing 100084, China \\ Correspondence should be addressed to Fuquan Zhao; zhaofuquan@tsinghua.edu.cn
}

Received 5 September 2021; Accepted 27 December 2021; Published 18 January 2022

Academic Editor: Seyed Ali Ghahari

Copyright (c) 2022 Zongwei Liu et al. This is an open access article distributed under the Creative Commons Attribution License, which permits unrestricted use, distribution, and reproduction in any medium, provided the original work is properly cited.

Intelligent connected vehicles (ICVs) have become the focus and development direction of the automobile industry. As a flexible intelligent terminal, ICVs will become a necessary part of the intelligent transportation system. The routes of developing ICVs based on "vehicle to X" (V2X) can effectively alleviate the demands of vehicles for intelligent functions and cut related research costs, accelerating commercialization of ICVs and leading to many social benefits. At present, China has made it clear to develop ICVs based on V2X, which requires simultaneous intelligent upgrades of vehicles and transportation infrastructure. Therefore, intelligent upgrades of transportation infrastructure must match the functional requirements of ICVs. In addition, the investment in intelligent upgrades of transportation infrastructure is mainly from the government, so the costs must be controlled reasonably to find the most cost-effective upgrade route. In this paper, the types of intelligent transportation infrastructures were determined by sorting out the demands of ICVs for transportation infrastructure, and the deployment methods and upgrade routes of intelligent transportation infrastructures were designed. Then, the cost evaluation model for intelligent upgrade of transportation infrastructures was established, based on which, the cost evaluation of different intelligent upgrade routes of transportation infrastructure was carried out in closed highway and open urban road scenarios to determine the optimal route. Besides, the key elements affecting the cost of transportation infrastructure upgrades were identified, and their impact degrees on transportation infrastructure upgraded were analyzed by scenario analysis. The results show that the intelligent transportation infrastructure for advanced ICVs mainly includes communication base stations, roadside units (RSUs), vision sensors, millimeter-wave radars, laser radars (LiDARs), meteorological sensors, intelligent signal machines, edge computing servers, and cloud computing centers. The route of deploying primary intelligent transportation infrastructure at first and then directly upgrading them to advanced level can well match the functional requirements of ICVs on the basis of lower costs. The costs of RSUs, LIDARS, and edge computing servers as well as data transmission rate of $5 \mathrm{G}$ are key elements affecting the costs of intelligent upgrades of transportation infrastructure.

\section{Introduction}

Intelligent connected vehicles (ICVs) based on the new generation of information and communication technology have become the recognized development direction of future vehicles all over the world $[1,2]$. Based on the core autonomous driving functions, ICVs can replace human beings to complete driving tasks. It can not only provide passengers with a safer, more comfortable, and intelligent driving experience but also improve travel efficiency [3-6], save energy and reduce emissions [7-9], and reduce the traffic accident rate [10-12]. At present, the development route of autonomous driving is gradually changing from vehicle intelligence to "vehicle to X" (V2X) [13]. In the past, some automobile and automobile-related industry giants tried to develop ICVs based on vehicle intelligence, but industry practice shows that the technical difficulty of realizing autonomous driving on this route is far beyond expectation, and the costs are high. As a result, there are increasingly more countries and enterprises attaching importance to the development route of V2X [14]. Among them, China, whose government has a stronger ability of 
resource coordination, is the most typical one. At present, it has made it clear that it will promote the coordinated construction of V2X with Chinese characteristics, that is, supporting and promoting the accelerated development of ICVs with intelligent transportation infrastructure.

In the mode of V2X, ICVs can share information with other connected vehicles as well as high-performance sensors and computing platforms at roadside through highspeed communication technologies such as $5 \mathrm{G}$ so as to realize multiple perception fusion and decision optimization. It can not only provide more comprehensive sensing information for vehicles to improve safety but also reduce performance requirements of vehicle hardware, enable redundancy, and reduce costs, which contributes to the improvement of the penetration rate of ICVs and the establishment of a new transportation system with higher efficiency and more convenience. Furthermore, it is also in line with the direction of intelligent upgrades of urban and transportation systems and will drive the upgrade of information infrastructure [15].

Therefore, the environment of intelligent transportation infrastructure is a key element supporting the future development of ICVs in China. The intelligent upgrade of transportation infrastructure should not only match the technical progress of ICVs to produce synergies but also be appropriately ahead of it and reserved to avoid repeated construction. Since cost performance evaluation is one of the significant elements to make decisions on the intelligent upgrade of transportation infrastructure, it is important to evaluate the upgrade cost of different development paths based on ICV compatibility of intelligent transportation infrastructure. At present, some of the scholars have elaborated on intelligent transportation infrastructure at the macrolevel [15-19], and others have optimized and innovated the deployment methods of them, such as communication environment $[20,21]$, roadside units (RSUs) [22-26], and sensors [22, 27-30]. However, there are few scholars analyzing the deployment costs of them.

Referring to the hierarchical definition of vehicle intelligence in China, intelligent transportation infrastructures were clarified by sorting out the requirements of different ICVs for them. On this basis, two typical scenarios were chosen, including open highways and closed urban roads, to evaluate the cost of intelligent transportation infrastructure through different deployment routes. In combination with the predictions of the market penetration rate and technical development level of ICVs at various levels, the optimal route for intelligent upgrade of transportation infrastructure was determined. Finally, some directional suggestions were put forward for the development of the ICV industry based on the research results.

\section{Setting of Intelligent Levels of Transportation Infrastructure}

2.1. Classification of Intelligent Levels of Vehicles. In the mode of V2X, the intelligent levels of vehicles should be evaluated from two aspects: automation level and connectivity level. As shown in Figure 1, referring to the classification of autonomous driving technology by the Society of Automotive Engineers and the classification of vehicle automation and connectivity levels as set forth in the Energy-Saving and New Energy Vehicle Technology Roadmap 2.0 released by China Society of Automotive Engineers in 2020 [31], vehicle intelligence is divided into three levels: primary, intermediate, and advanced levels, each of which corresponds to a certain level of automation and connectivity.

\subsection{Requirements of ICVs for Transportation Infrastructure.} Considering the functions that need to be realized in automation and connectivity of ICVs, the functional requirements of ICVs for transportation infrastructure are divided into five aspects: communication guarantee, information transmission, safety warning, driving assistance, and extended services [16].

In terms of communication guarantee, the data transmission rate of a primary ICV in the mode of V2X is lower than $100 \mathrm{Kbps}$, and the communication delay should be controlled within $100 \mathrm{~ms}$. Only the LTE communication network can meet the demand. As for an intermediate ICV, the data transmission rate is bigger than $10 \mathrm{Mbps}$, and the communication delay needs to be controlled between 20 and $100 \mathrm{~ms}$. LTE communication network can no longer meet the demand, and only the 5G-NR communication network is able to guarantee the data transmission rate. As for an advanced ICV, the data transmission rate is bigger than $100 \mathrm{Mbps}$, and the communication delay must be strictly controlled within $20 \mathrm{~ms}$, which can be guaranteed only by a complete 5G-NR communication network.

In terms of information delivery, the primary ICVs, on the one hand, are able to get real-time traffic and road condition information through RSUs. On the other hand, they can obtain relevant location and status information for communication with service areas. On the basis of primary ICVs, intermediate intelligent vehicles require RSUs to provide state information of nearby traffic participants to assist decision-making and update dynamic HD maps in real time. On the basis of primary and intermediate intelligent vehicles, advanced intelligent vehicles need to communicate with all urban intelligent terminals through the Internet of Things so as to realize unified vehicle scheduling and platooning.

In terms of safety warning, primary ICVs can realize real-time monitoring of illegal behaviors through RSUs and roadside sensors. Intermediate ICVs need real-time dangerous driving warning and vehicle violation warning through RSUs and roadside sensors. Advanced ICVs need real-time information sharing to avoid risks autonomously.

In terms of driving assistance, primary ICVs require intelligent transportation infrastructure to provide auxiliary perception information. In addition to auxiliary perception requirements, intermediate ICVs also need roadside edge computing servers to provide partial support of calculation and decision-making. Advanced ICVs are bound to face more complex driving tasks, requiring more efficient intelligent transportation infrastructure to assist in vehicle perception, decision-making, and control. 


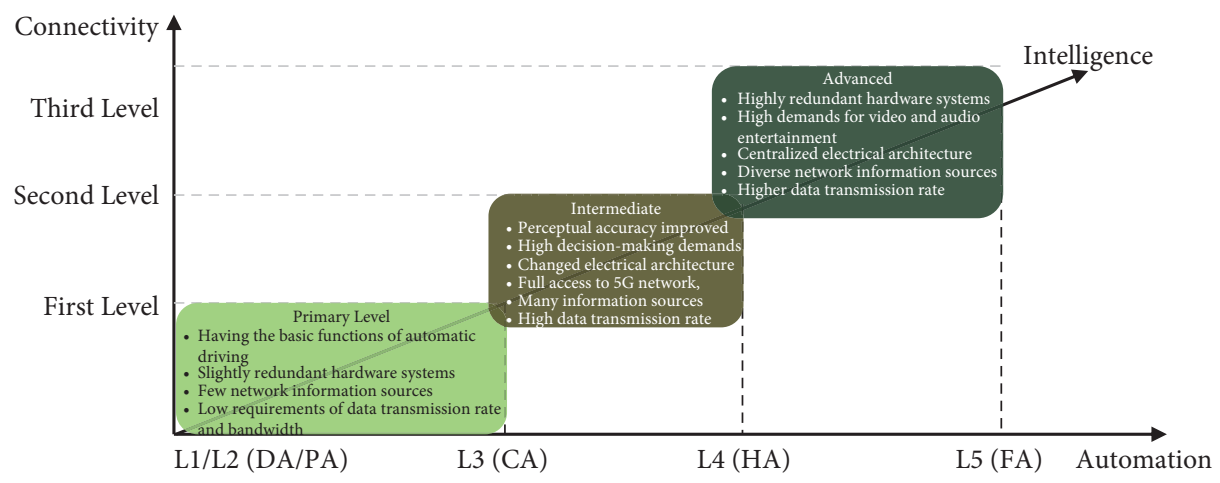

Figure 1: Classification of intelligent levels of vehicles.

In terms of service extension, intelligent transportation infrastructure should support sharing among intelligent vehicles, smart energy services, and so on.

\subsection{Classification of Intelligent Levels of Transportation Infrastructure. According to the functional requirements analyzed above, the intelligent transportation infrastructure is divided into three levels, primary, intermediate, and ad- vanced levels, as shown in Table 1.}

\subsection{Architecture of Intelligent Transportation Infrastructure} under Ideal Conditions. Ideally, intelligent transportation infrastructure can be divided into four layers, as shown in Figure 2: traffic participants, sensors/actuators, edge computing servers, and cloud computing centers. In the data collection stage, data generated by people, vehicles, and roads in traffic activities are collected by sensors such as vision sensors, LiDARs, and millimeter-wave radars, transmitted to all edge computing servers for immediate processing, and finally collected to the cloud computing centers. After data analysis is completed in cloud computing centers, execution instructions are transmitted along the path of "cloud computing center-edge computing serveractuator-traffic participant," ultimately affecting the individual terminal directly. In this process, communication base stations provide a reliable communication environment for information exchange between all levels of infrastructure so as to truly realize the real-time acquisition and transmission of data as well as update and delivery of instructions.

\section{Cost Evaluation Model for Intelligent Upgrade of Transportation Infrastructure}

3.1. Key Assumptions of the Model. The cost evaluation model of intelligent upgrade of transportation infrastructure is built based on the following three assumptions:

(1) Due to the possible emergence of new intelligent transportation infrastructure in the future, the costs of intelligent upgrade will be evaluated based on the transportation infrastructure that has been massproduced or has not been mass-produced but successfully developed; that is, the demand for improving intelligent levels could be met by increasing the number of related known facilities deployed.

(2) Advanced intelligent transportation infrastructure can provide ICVs with integrated services of perception, decision-making, and control. Sensors with stronger sensing ability and anti-interference ability are required. Therefore, it is assumed that the cost of sensors for primary and intermediate intelligent transportation infrastructure refers to the current market price while that of advanced intelligent transportation infrastructure would increase by $20 \%$ based on the current market price.

(3) Intelligent transportation infrastructure is a combination of hardware and software, and the possible trend is that the importance of software will grow. However, the iteration cycle of software is short, and the cost is often lower than that of hardware and usually directly included in hardware systems. Therefore, only the cost of hardware for transportation infrastructure is evaluated. In addition, it is difficult to estimate the medium cost usually regarded as fixed expenditure, such as human input, in the process of deployment and operation management of transportation infrastructure, which will not be considered.

(4) The change of penetration rates of ICVs at different levels exerts a direct impact on the demand for intelligent transportation infrastructure. The aim of this paper is to compare the maximum construction costs required to upgrade intelligent transportation infrastructure at different levels. Therefore, it is assumed that the penetration rate of ICVs at each level is $100 \%$ in the corresponding scenario.

3.2. Model Construction. In this part, the framework of the cost evaluation model is built, based on which the research work is gradually carried out. As shown in Figure 3, firstly, the required functions of road traffic infrastructure are inferred based on the intelligent levels of ICVs, based on which the corresponding levels of intelligent transportation infrastructure are set. Secondly, the plan for deploying different levels of intelligent transportation infrastructure 
TABLE 1: Classification of intelligent levels of transportation infrastructure.

Intelligent levels of transportation infrastructure

Primary

Shares key information with ICVs and replaces them to complete part of the perception work

(1) Provides ICVs with information on traffic signals, real-time traffic flows, and other road conditions based on vehicle-road-cloud communication

(2) Provides traffic management and law enforcement departments with road information, such as traffic accidents and violations of laws and regulations, based on road sensors

(3) Provides ICVs with information of gas stations, parking lots, and other peripheral service facilities based on the network of transportation auxiliary infrastructure

(1) Provides real-time point-to-point information of surrounding roads and helps ICVs compute and make decisions based on vehicle-road-cloud-people

Replaces the ICVs to complete all perception work Intermediate and shares a part of the computing decision-making work through edge computing servers communication and high-precision GPS positioning (2) Provides traffic participants with more peripheral service information at a faster pace with the gradual expansion of the coverage of the $5 \mathrm{G}$ communication environment

(1) Controls traffic participants in a cooperative manner and makes all decisions for ICVs based on the communication among vehicles, roads, clouds, people, buildings, and intelligent devices

Advanced

Improves computing ability of ICVs and connect

(2) Smoothly provides ICVs with more diversified services, information, and more functions such as photovoltaic wireless charging

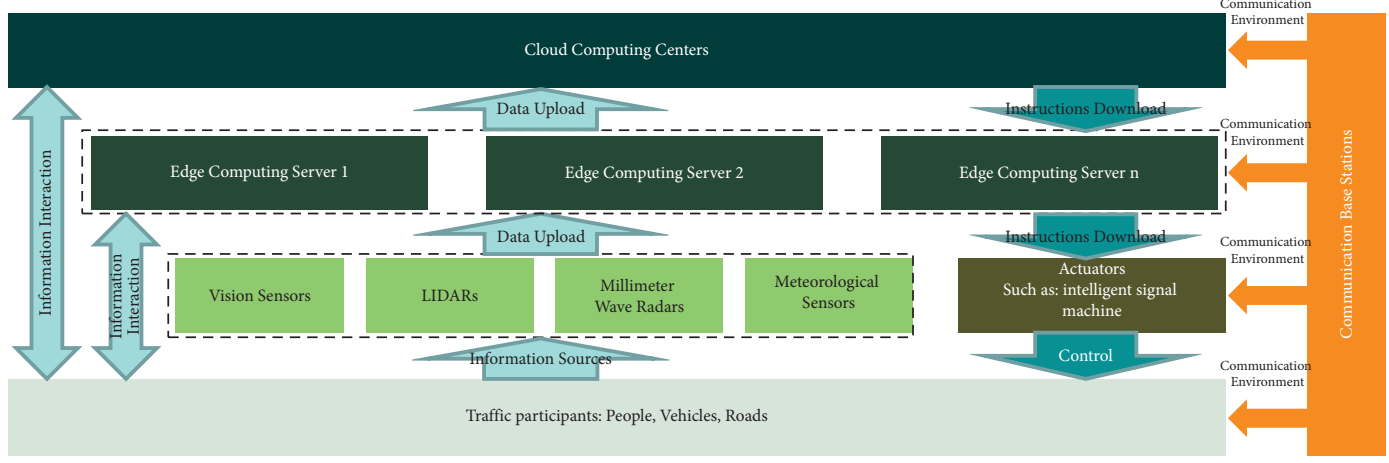

Figure 2: Architecture of intelligent transportation infrastructure under ideal conditions.

(namely, the types and quantities of specific facilities) under different scenarios is determined, and the corresponding one-time upgrade cost is calculated based on the coverage of various intelligent transportation infrastructure and combined with the transmission rate of road network data. Finally, the costs of four different routes to complete the deployment of advanced intelligent transportation infrastructure are evaluated, and as a result, suggestions for intelligent upgrade of transportation infrastructure are given in combination with the actual situation of the industry.

As for specific application scenarios, closed highways with large traffic flow and open roads in typical large cities in China are selected for evaluation. On the one hand, compared with other scenarios, these two scenarios are more representative and complex, contributing to greater benefits if the intelligent upgrade of corresponding transportation infrastructure is realized. On the other hand, they are also the two scenarios that local governments in China focus on to promote the development of ICVs and intelligent transportation systems. In addition, these two scenarios correspond to the standard of Chinese road classification. Among them, Beijing-Zhangjiakou Highway is selected as a closed highway scenario, while the entire road network within the Fifth Ring Road of Beijing is selected as an open road scenario. Tables 2 and 3 show the specific attribute parameters of the two evaluation objects. 


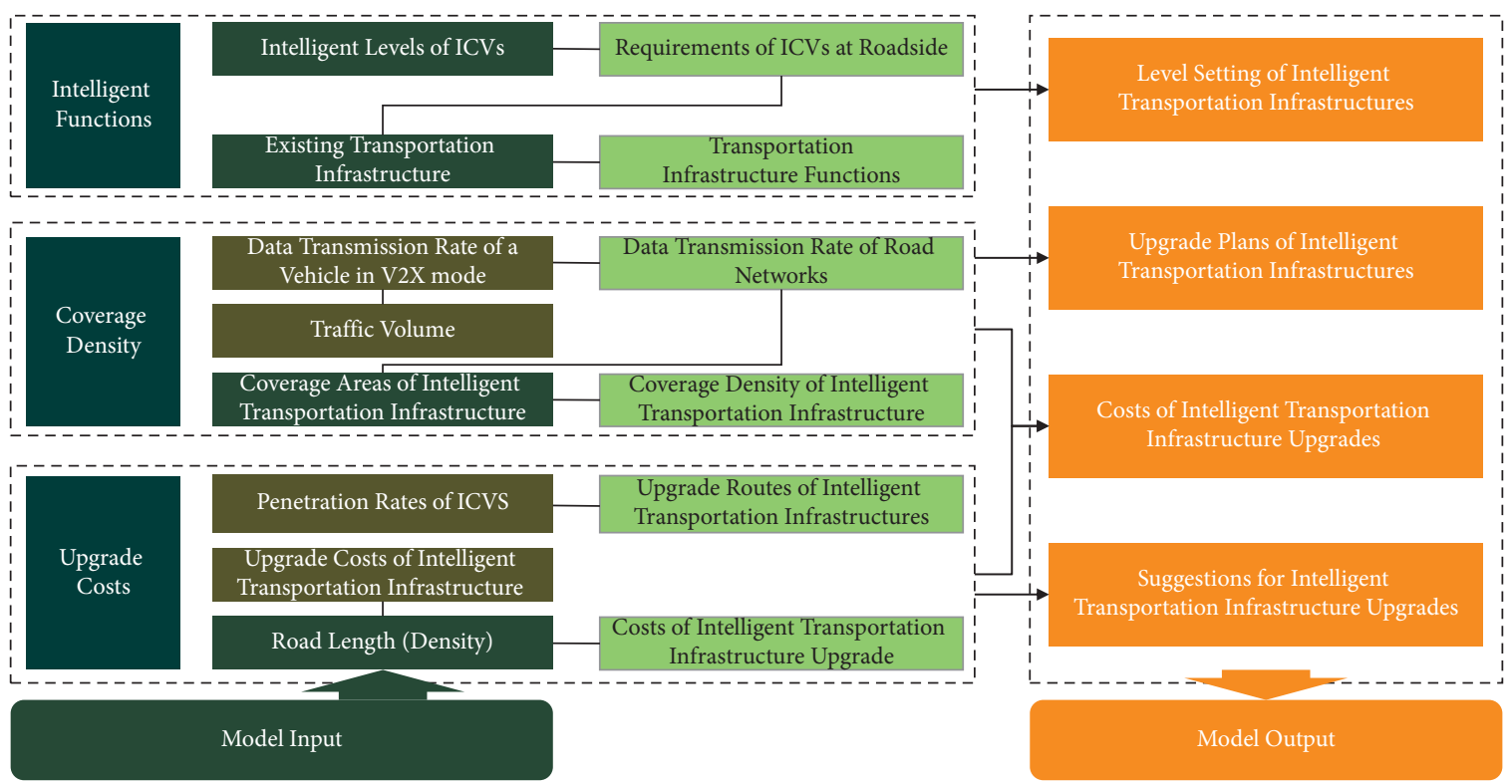

Figure 3: Cost evaluation model for intelligent upgrade of transportation infrastructure.

TABle 2: Attribute parameters of the closed highway scenario.

\begin{tabular}{lc}
\hline Parameter & Value \\
\hline Road length $(\mathrm{km})$ & 148.2 \\
Traffic flow in one lane (number of vehicles $/ \mathrm{m})$ & 0.02 \\
Number of lanes (two directions) & 6 \\
The year construction started & 1998 \\
Investment & $¥ 3,000,000,000$ \\
\hline
\end{tabular}

TABle 3: Attribute parameters of the open urban road scenario.

\begin{tabular}{lc}
\hline Parameter & Value \\
\hline Road length $(\mathrm{km})$ & $3,728.53$ \\
Traffic flow in one lane (number of vehicles $/ \mathrm{m})$ & 0.1 \\
Number of lanes (two directions) & 4 \\
Road density $\left(\mathrm{km} / \mathrm{km}^{2}\right)$ & 5.59 \\
Average density of road intersections (number of vehicles $/ \mathrm{km})$ & 2.3 \\
\hline
\end{tabular}

3.3. Model Inputs. Based on the analysis above of intelligent function requirements of traffic infrastructure, the main traffic infrastructure involved is determined to include LTE base stations, 5G base stations, RSUs, vision sensors, millimeter-wave radars, LiDARs, intelligent signals, meteorological sensors, edge computing servers, and cloud computing centers. As shown in Table 4, currently available or forthcoming transportation infrastructure with relatively good performance is selected as the model input. Its functional parameters and costs can be found in e-commerce platforms, the manufacturers' websites, product manuals, or research reports.

After the types of transportation infrastructure products are determined, the demand for various products per kilometer is calculated for different intelligent levels of transportation infrastructure.
The communication base stations are used to build communication network so as to provide communication environment for ICVs and intelligent transportation infrastructure. LTE or $5 \mathrm{G}$ communication base stations need to be deployed to meet both requirements of full coverage and data transmission rates. $i$ represents a closed highway or an open urban road when it is equal to 1 or 2 . On closed highways, the demand per kilometer for the communication base stations to fully cover the roads $d_{\text {com,1 }}$ can be calculated by equation (1), where $r_{\text {com }}$ is the coverage radius of base stations.

$$
d_{\mathrm{com}, 1}=\frac{1}{2 r_{\mathrm{com}}} .
$$

On open urban roads, the deployment of communication base stations to meet requirements of full coverage is 
TABLE 4: Performance parameters and costs of intelligent transportation infrastructure.

\begin{tabular}{|c|c|c|c|}
\hline Facility & Product & Performance or cost/unit & Value \\
\hline \multirow{3}{*}{ LTE base station } & \multirow{3}{*}{ Zte BS8912 } & Coverage radius $/ \mathrm{km}$ & 1 \\
\hline & & Transmission rate/Mbps & 110 \\
\hline & & Cost/CNY(¥) & 3,000 \\
\hline \multirow{3}{*}{$5 \mathrm{G}$ base station } & \multirow{3}{*}{$\begin{array}{l}\text { No specific product information, estimated according to } \\
\text { relevant literature }\end{array}$} & Coverage radius $/ \mathrm{km}$ & 0.2 \\
\hline & & Transmission rate/Mbps & 1 \\
\hline & & Cost/CNY(¥) & 30,000 \\
\hline \multirow{2}{*}{ RSU } & \multirow{2}{*}{ Datang Telecom DTVL3100-RSU } & Coverage radius $/ \mathrm{km}$ & 1 \\
\hline & & Cost/CNY(¥) & 70,000 \\
\hline \multirow{3}{*}{ Vision sensor } & \multirow{3}{*}{ Haikang cd7087f/DS - $2 \mathrm{~V}$} & Coverage radius $/ \mathrm{km}$ & 0.2 \\
\hline & & Primary and intermediate costs/CNY(¥) & 4,630 \\
\hline & & Advanced cost/CNY(¥) & 5,556 \\
\hline \multirow{3}{*}{ Millimeter-wave radar } & \multirow{3}{*}{ Continent ARS $408-2177 \mathrm{GHz}$} & Coverage radius $/ \mathrm{km}$ & 0.25 \\
\hline & & Primary and intermediate costs/CNY(¥) & 3,500 \\
\hline & & Advanced cost/CNY(¥) & 4,200 \\
\hline \multirow{3}{*}{ LiDAR } & \multirow{3}{*}{ Velodyne VLP-32C } & Coverage $/ \mathrm{km}$ & 0.2 \\
\hline & & Primary and intermediate costs/CNY(¥) & 203,667 \\
\hline & & Advanced cost/CNY(¥) & 244,400 \\
\hline $\begin{array}{l}\text { Intelligent signal } \\
\text { machine }\end{array}$ & Hisense SC3101 & Primary and intermediate costs/CNY(¥) & 46,000 \\
\hline \multirow{3}{*}{ Meteorological sensor } & \multirow{3}{*}{ TRM-ZS7 } & $\begin{array}{l}\text { Deployment density on highways/ } \\
\text { number } / \mathrm{km}\end{array}$ & 0.067 \\
\hline & & $\begin{array}{l}\text { Deployment density on urban roads/ } \\
\text { number } / \mathrm{km}^{2}\end{array}$ & 0.33 \\
\hline & & Cost/CNY(¥) & 75,000 \\
\hline \multirow{3}{*}{ Edge computing server } & \multirow{3}{*}{$\begin{array}{l}\text { No specific product information, referring to research } \\
\text { reports }\end{array}$} & $\begin{array}{l}\text { Deployment density on highways/ } \\
\text { number } / \mathrm{km}\end{array}$ & 4 \\
\hline & & $\begin{array}{l}\text { Deployment density on urban roads/ } \\
\text { number/cross }\end{array}$ & 2 \\
\hline & & Cost/CNY $(¥)$ & 175,000 \\
\hline \multirow{3}{*}{$\begin{array}{l}\text { Cloud computing } \\
\text { center }\end{array}$} & \multirow{3}{*}{$\begin{array}{l}\text { No specific product information, referring to research } \\
\text { reports }\end{array}$} & $\begin{array}{c}\text { Deployment density on highways/ } \\
\text { number } / \mathrm{km}\end{array}$ & 0.01 \\
\hline & & $\begin{array}{l}\text { Deployment density on urban roads/ } \\
\text { number/km }\end{array}$ & 0.025 \\
\hline & & Cost/CNY(¥) & $20,000,000$ \\
\hline
\end{tabular}

shown in Figures 4 and 5, respectively. Squares surrounded by black borders in both of the figures represent urban areas of $1 \mathrm{~km}^{2}$, while circles (including full circles, semicircles, and quarter circles) represent areas covered by communication stations. In Figure 4, four gray quarter circles (1, 2, 3, and 4) and a cyan full circle (5) cover an urban area of $4 \mathrm{~km}^{2}$ $(2 \mathrm{~km} \times 2 \mathrm{~km})$. Each urban area of $1 \mathrm{~km}^{2}$ needs to be covered by two-quarter circles. Similarly, in the left picture of Figure 5 , four full circles $(5,6,8$, and 9$)$, four semicircles $(2,3,4$, and 7), and a quarter circle (1) in dark green cover the square urban area of $1 \mathrm{~km}^{2}$ for the first time. Then in the right picture of Figure 5, the blank left by the first coverage was filled by the four full circles $(10,11,13$, and 14), four semicircles $(12,15,16$, and 17), and a quarter circle (18) in cyan. Combined with the road density within the Fifth Ring Road of Beijing, the demand per kilometer in different scenarios for communication base stations to realize full coverage $d_{\text {com, } 2}$ can be worked out by equation (2), where $n_{q c}, n_{s c}$, and $n_{f c}$ represent the number of quarter circles, semicircles, and full circles in square areas separately and $\rho$ represents the road density.

$$
d_{\mathrm{com}, 2}=\frac{0.25 n_{q c}+0.5 n_{s c}+n_{f c}}{\rho} .
$$

Then, whether the requirements on data transmission rates are met has to be verified. If not, the number of base stations should be added on the basis of the requirements of full coverage until the requirements of data transmission rates are met. The base station increment per kilometer $\Delta d_{\text {com }, i}$ can be calculated by equation (3), where $C_{\text {demand }}$ represents the ideal data transmission rate and $w_{\text {com }}$ represents the data transmission rate of a base station.

$$
\Delta d_{\mathrm{com}, i}=\frac{C_{\mathrm{demand}}-w_{\mathrm{com}} d_{\mathrm{com}, i}}{w_{\mathrm{com}}}, i=1,2 .
$$

The calculation above shows that, on closed highways, as for the primary intelligent transportation infrastructure, LTE base stations with full coverage can meet both of the requirements. On open urban roads, however, the number of LTE base stations should be increased. In two different scenarios above, as for the intermediate and advanced intelligent transportation infrastructure, 5G base stations 


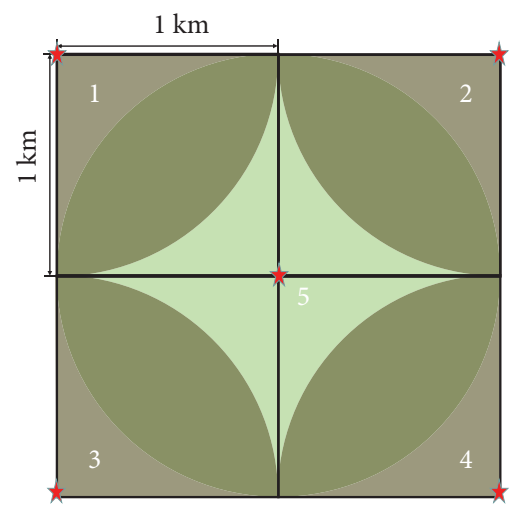

FIGURE 4: Deployment of LTE base stations on open urban roads.
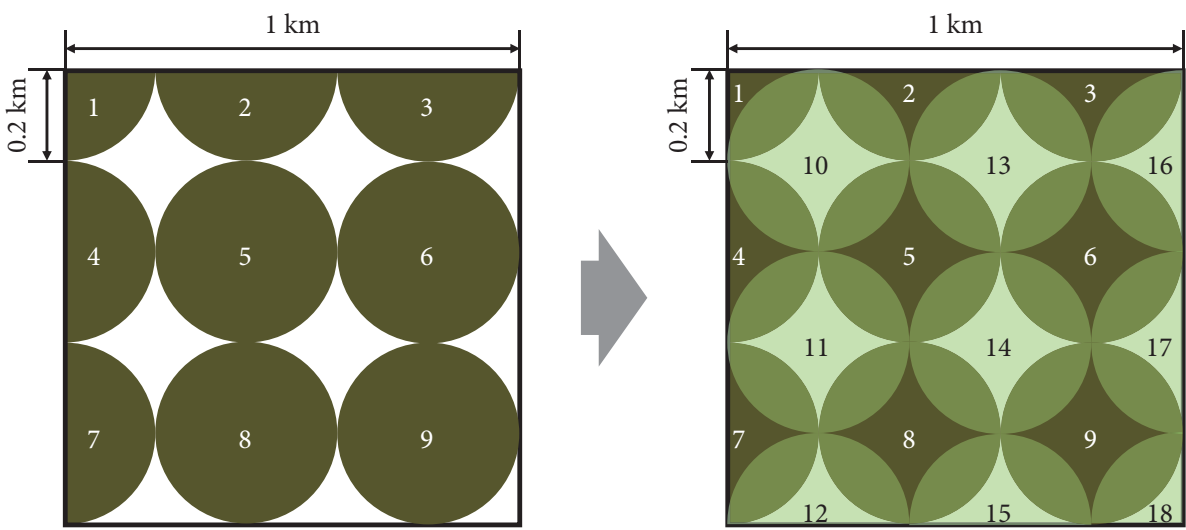

FIGURE 5: Deployment of 5G base stations on open urban roads.

instead of LTE base stations must be used to meet the requirements of data transmission rate. A possible upgrade route is clearly indicated then.

Sensors are the "eyes" of smart roads, sensing vehicle behaviors, traffic flows, emergencies, and even weather conditions in real time. The demand per kilometer for visual sensors $d_{\text {cam }, i}$ and millimeter-wave radars $d_{m w r, i}$ in different road scenarios can be uniformly calculated by equation (4), where $r_{\text {cam }}$ and $r_{\text {mwr }}$ represent the coverage radius of vision sensors and millimeter-wave radars.

$$
\begin{aligned}
& d_{\mathrm{cam}, i}=2 \cdot \frac{1}{r_{\mathrm{cam}}}, \\
& d_{m w r, i}=2 \cdot \frac{1}{r_{m w r}}, \quad i=1,2 .
\end{aligned}
$$

LiDAR selected in this paper is a mechanical rotatable one, whose coverage area is a circle with the radar as the center and the coverage radius as the coverage distance. Therefore, the demand per kilometer for LiDAR in different road scenarios $d_{L i, i}$ can be calculated by equation (5), where $r_{l i}$ is the coverage radius of LiDAR.

$$
d_{l i, i}=2 \cdot \frac{1}{2 r_{l i}}, \quad i=1,2 .
$$

The coverage range of meteorological sensors refers to the Chinese national standard [32]. As for closed highways, the deployment spacing should not be greater than $15 \mathrm{~km}$, which means the demand for meteorological sensors is 0.067 units per kilometer. As for open urban roads, its coverage area should not be bigger than $3 \mathrm{~km}^{2}$, which means that the coverage radius is about $1 \mathrm{~km}$. As a result, its deployment scheme is basically the same as that of LTE base stations in Figure 3, with a demand $d_{w t, 2}$ of 0.089 units per kilometer.

Intelligent signal machines are mainly deployed at urban intersections. Under the control of edge or central cloud control platforms, it can optimize signal timing in real time according to the optimization algorithm of traffic flows and road networks. The signal status information can also be sent to ICVs to help them pass through intersections more efficiently. In general, only one intelligent signal machine is deployed at each intersection. Combined with the road density within the Fifth Ring Road of Beijing, the demand for intelligent signal machines per kilometer of urban roads $d_{s i, 2}$ is 2.3 units.

RSU is a communication gateway deployed at the roadside, which is responsible for data exchange between the ICVs, roads, and clouds. Sensors can upload data to cloud control platforms of intelligent transportation through RSUs. Therefore, in order to eliminate the perceptual blind area of roads, the number of RSUs should be the same as that of the type of sensors with the highest deployment density. 
On closed highways, RSUs are mainly used to connect sensors to the network. Its demand per kilometer $d_{R S U, 1}$ can be calculated according to the following equation:

$$
d_{R S U, 1}=\max \left\{d_{c a m, 1}, d_{m w r, 1}, d_{l i, 1}, d_{w t, 1}\right\} .
$$

On open urban roads, RSUs are used to connect sensors and intelligent signal machines to the network at the same time. Its demand per kilometer $d_{R S U, 2}$ can be calculated according to the following equation:

$$
d_{R S U, 2}=\max \left\{d_{c a m, 2}, d_{m w r, 2}, d_{l i, 2}, d_{w t, 2}\right\}+d_{s i, 2} .
$$

Cloud control platforms of intelligent transportation are composed of basic cloud control platforms and collaborative applications. Collaborative applications mainly include control algorithms related to ICVs and intelligent transportation, while the basic cloud control platforms consist of edge computing servers and cloud computing centers, providing data, communication, and runtime environment for all kinds of collaborative applications. Therefore, the basic cloud control platforms are key components of the cyber-physical systems of intelligent transportation [16]. Due to the difficulty in obtaining pricing and functional parameters for edge computing servers and cloud computing centers, the viewpoints of several research reports are referred to $[33,34]$. On closed highways, the demand for computing servers $d_{M E C, 1}$ and cloud computing centers $d_{c t r, 1}$ per kilometer is 4 units and 0.01 units, respectively. On open urban roads, the demand for computing servers $d_{M E C, 2}$ and cloud computing centers $d_{c t r, 2}$ per kilometer is 2 units and 0.025 units. As primary intelligent transportation infrastructure is only used to provide ICVs with simple information of traffic and related services, the demand for edge computing and cloud computing servers is not urgent. However, intermediate and advanced intelligent transportation infrastructure are in urgent need of edge computing servers and cloud computing centers because they are used to provide sensing and decision-making services for ICVs and even used to optimize the traffic conditions of road networks, which requires edge computing servers and cloud computing centers. Therefore, as for primary intelligent transportation infrastructure, there is probably no need to deploy cloud computing centers, and the demand for edge computing servers should be multiplied by the sum of current market penetration rates of intermediate and advanced ICVs in China [35].

Finally, by multiplying the demand per kilometer for each type of transportation infrastructure product at different intelligent levels and then adding them together, the costs required to complete the corresponding deployment at one time can be calculated.

\section{Evaluation of the Cost of Intelligent Upgrades of Transportation Infrastructure}

\subsection{Evaluation of the Cost of Different Intelligent Upgrade Routes for Transportation Infrastructure}

4.1.1. Cost of One-Time Intelligent Upgrade of Transportation Infrastructure. In the actual upgrade process, it is unnecessary to obey the order of intelligent upgrade of primary, intermediate, and advanced infrastructure. Instead, a certain level of transportation infrastructure can be directly deployed ignoring some levels before it. Let $R(l, m, n, i)$ represent different construction routes, where $i$ represents closed highways or open urban roads when it is equal to 1 or 2, respectively. Besides, $l, m, n \in\{1,2,3\}$ represents the intelligent levels of transportation infrastructure at the first, second, and third construction stages, and numbers 1, 2, and 3 refer to primary, intermediate, and advanced intelligent infrastructure. For instance, $R(1,3,3,1)$ represents the route of open urban roads in which primary transportation infrastructure is built in the first place and then directly upgraded to the advanced level.

Using the model established above, the one-time deployment costs per kilometer of primary, intermediate, and advanced intelligent transportation infrastructure under different scenarios are calculated. On closed highways, the one-time construction costs per kilometer of primary, intermediate, or advanced intelligent transportation infrastructure are $¥ 895,880, ¥ 2,772,633$, and $¥ 3,276,160$, respectively, as shown in Figure 6. The device with the highest deployment cost among primary intelligent transportation infrastructure is RSU, whose cost takes about 78\% of the total. This is due to its high price and rigid and big demand for sensors. The device with the highest deployment cost among intermediate and advanced intelligent transportation infrastructure is LiDAR, whose cost takes about $36 \%$ and $37 \%$ of the total separately. This is because the intermediate and advanced ICVs have urgent demands for the roadside perception abilities and $\mathrm{HD}$ maps, so that LiDARs with higher perception accuracy and robustness are needed to cover the whole roads. Meanwhile, the costs of RSUs and edge computing servers are also high, taking about $25 \%$ and $21 \%$, respectively.

On open urban roads, the one-time construction costs per kilometer of primary, intermediate, or advanced intelligent transportation infrastructure are $¥ 914,441, ¥ 4,428,008$, and $¥ 5,913,908$, respectively, as shown in Figure 7. Similar to the situation of closed highways, the device with the highest deployment cost of primary intelligent transportation infrastructure is RSU, whose cost takes approximately $83 \%$ of the total. As for intermediate and advanced intelligent transportation infrastructure, the devices with the highest, second highest, and third highest costs are LiDARs, RSUs, and edge computing servers, respectively, taking about $44 \%$ and $40 \%, 19 \%$ and $15 \%$, and $18 \%$ and $14 \%$ of the total in order.

It is found that the deployment costs per kilometer of primary, intermediate, and advanced intelligent transportation infrastructure on open urban roads are $¥ 18,561$, $¥ 1,655,375$, and $¥ 2,637,748$ higher than that those on closed highways, respectively. On the one hand, the geometric configuration of urban roads is network structure whose road density is much higher than that of closed highways; on the other hand, traffic volume of urban roads is much bigger than that of closed highways. Therefore, there is a higher demand for communication base stations, meteorological sensors, edge computing servers, cloud computing centers, and RSUs. In addition, intermediate and advanced 


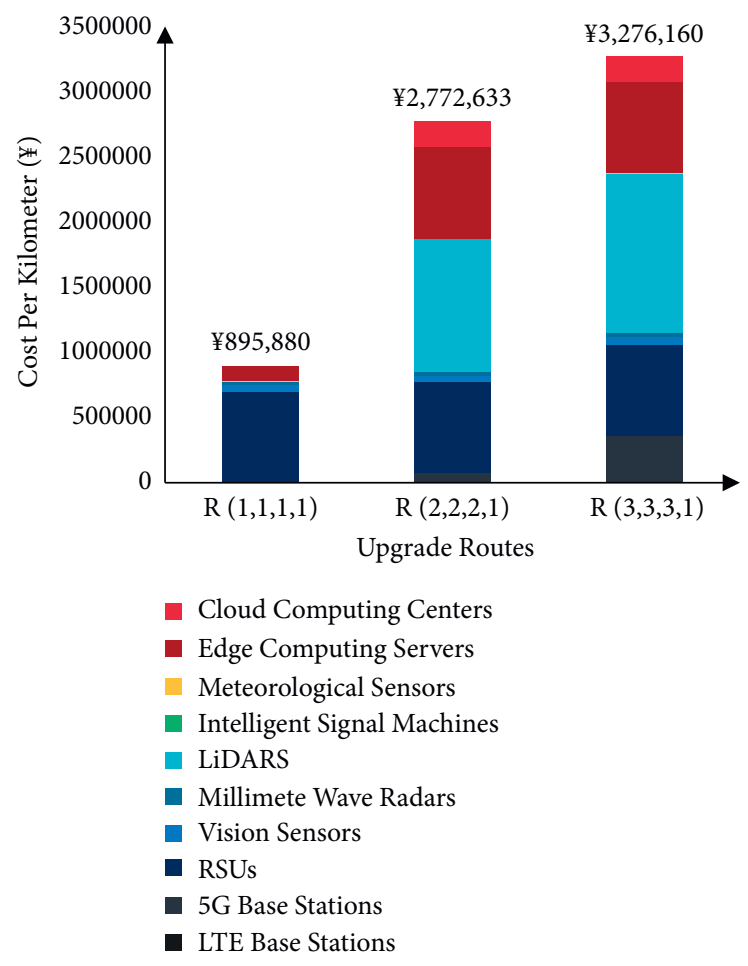

FIGURE 6: Cost of one-time upgrade of intelligent transportation infrastructure on closed highways.

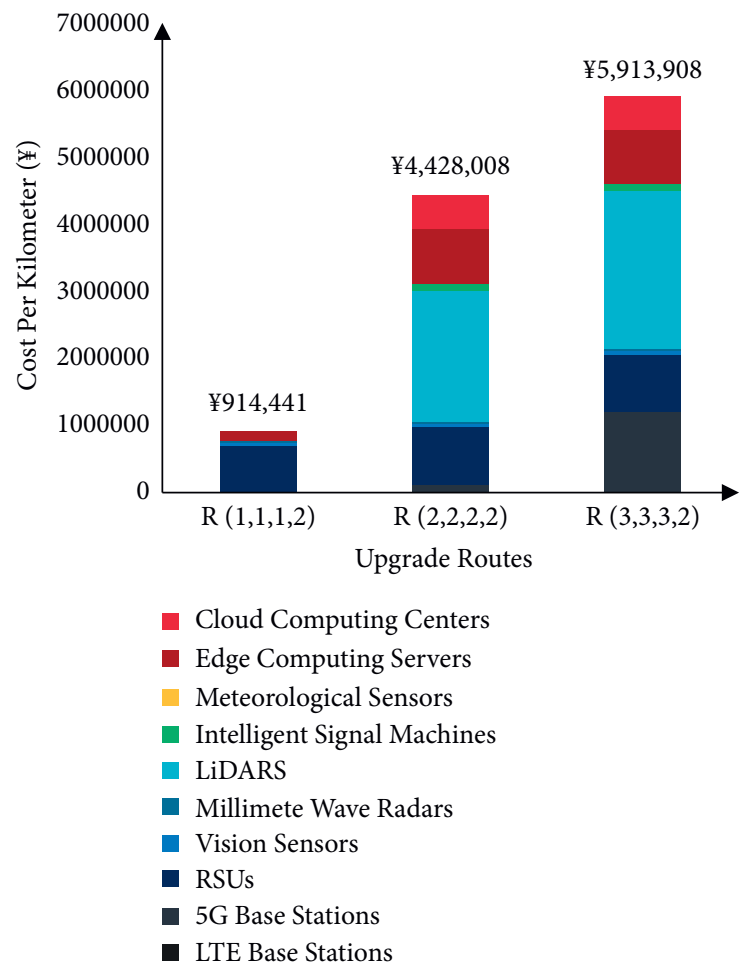

FiguRE 7: Cost of one-time upgrade of intelligent transportation infrastructure on open urban roads. 
intelligent transportation infrastructure requires intelligent signal machines at intersections on open urban roads, but not on closed highways.

\subsubsection{Evaluation of the Cost of Different Intelligent Upgrade} Routes for Transportation Infrastructure. Transportation infrastructure should be upgraded to the advanced level eventually to meet the demands of advanced ICVs, so there are four possible upgrade routes, as shown in Figure 8, which need upgrades one to three times.

$R(1,2,3, i)$ represents the route in which infrastructure is sequentially upgraded from primary to intermediate to advanced level. When primary intelligent infrastructure is upgraded to an intermediate one, RSUs and sensors can continue to be used. But in order to satisfy intermediate ICVs on perception, decision-making, communication, and other higher requests, LTE base stations should be replaced by $5 \mathrm{G}$ base stations and LiDARs, and intelligent signal machines and cloud computing centers should be deployed. Besides, the number of edge computing servers should be increased. When intermediate intelligent infrastructure is upgraded to an advanced one, existing $5 \mathrm{G}$ base stations, RSUs, intelligent signal machines, meteorological sensors, edge computing servers, and cloud computing centers can continue to be used, but there are necessary needs to add 5G base stations and replace exiting sensors with those that have higher performance. So, the costs of single-stage deployment on the step-by-step upgrade route are equal to the costs of the incremental components of the intelligent transportation infrastructure between two stages, which are less than the deployment costs of a one-time upgrade to the corresponding level. There are similar situations existing in $R(1,3,3, i)$ and $R(2,3,3, i)$, which are separately considered in corresponding cost evaluations.

Figures 9 and 10 depict the total costs per kilometer of different upgrade routes on closed highway and open urban road scenarios, respectively. On closed highways, the costs of $R(1,2,3,1), R(1,3,3,1), R(2,3,3,1)$, and $R(3,3,3,1)$ are $¥ 4370293, ¥ 3351960, ¥ 4368793$, and $¥ 3276160$. Compared with $R(3,3,3,1), R(1,2,3,1), R(1,3,3,1)$, and $R(2,3,3,1)$ need additional costs of $33.4 \%, 2.3 \%$, and $33.3 \%$ separately. On open urban roads, the costs of $R(1,2,3,2), R(1,3,3,2), R$ $(2,3,3,2)$, and $R(3,3,3,2)$ are $¥ 7,944,499$, $¥ 5,989,299$, $¥ 7,943,408$, and $¥ 5,913,908$. Compared with $R(3,3,3,2), R$ $(1,2,3,2), R(1,3,3,2)$, and $R(2,3,3,2)$ need additional costs of $34.3 \%, 1.3 \%$, and $34.3 \%$ separately.

What is more, a comparison was made between the cost evaluation results above and the costs of ordinary roads (replaced by investment amount). As for closed highways, Beijing-Zhangjiakou Highway spanning 148 kilometers began to be built at the end of 1998, which was invested $¥ 3,000,000,000$ then. Considering the inflation of RMB from 1999 to 2020, the total investment now is about $¥ 4,680,000,000$, that is, $¥ 31,621,622$ per kilometer. It is concluded that for Beijing-Zhangjiakou Highway, the intelligent upgrade cost per kilometer is only $13.8 \%$ of the original construction cost per kilometer, even if the route with the highest costs is chosen.

As for open urban roads, it is difficult to estimate the construction costs per kilometer of urban roads due to the differences among different cities in land prices, labor costs, geographical features, and construction plans. However, because the prices of urban land are generally much higher than those of the suburban areas through which the highway passes, and the complex urban traffic conditions have high requirements for the advanced and complete degree of infrastructure, it is inferred that the cost per kilometer of urban roads is probably higher than that of highways. In addition, the deployment of intelligent transportation infrastructure on open city roads is expected to bring higher benefits since traffic volume there is far greater than that on highways.

All in all, although intelligent upgrade of transportation infrastructure leads to higher costs, it is far from being difficult to bear the burden. In other words, the V2X mode is cost-feasible at the roadside.

4.1.3. Selection of Intelligent Upgrade Routes for Transportation Infrastructure. As is shown above, there is no doubt that $R(3,3,3, i)$ is the route with the lowest costs. But in practice, the feasibility of one-step deployment is very low. Firstly, advanced ICVs are still under research and development, and their market penetration rate is almost zero, so the payback and accumulation of advanced intelligent transportation infrastructure would be limited. Secondly, replacement is necessary when service time is beyond infrastructure life, so advanced deployment is likely to lead to serious waste. Thirdly, current advanced ICVs and transportation infrastructure are still of much uncertainty. Driven by technological progress and business model innovation, new transportation infrastructure products with higher cost performance may appear in the future. As a result, it is not appropriate to directly deploy advanced intelligent transportation infrastructure based on existing products.

Taking into account the costs of intelligent upgrading of transportation infrastructure and the expected market penetration rates of ICVs at different levels, it is concluded that $R(1,3,3, i)$ is likely to be the best choice at present. First of all, it has a cost advantage. Costs of $R(1,3,3,1)$ and $R(1,3$, $3,2)$ are only $2.3 \%$ and $1.3 \%$ higher than $R(3,3,3,1)$ and $R$ $(3,3,3,2)$, respectively, lower than $R(1,2,3, i)$ and $R(2,3$, $3, i)$. Next, at present, intermediate ICVs are likely to play only a continuing role in the technological transition stage. In fact, under specific application scenarios, there is no essential difference between L4 and L5autonomous driving, which means that advanced ICVs will also be qualified for mass production when intermediate ICVs are fully industrialized. At last, both intermediate and advanced ICVs need a 5G communication environment, and advanced intelligent transportation infrastructure can meet the demands of intermediate and advanced ICVs. Therefore, it is reasonable to deploy intelligent infrastructure ignoring the intermediate stage. 


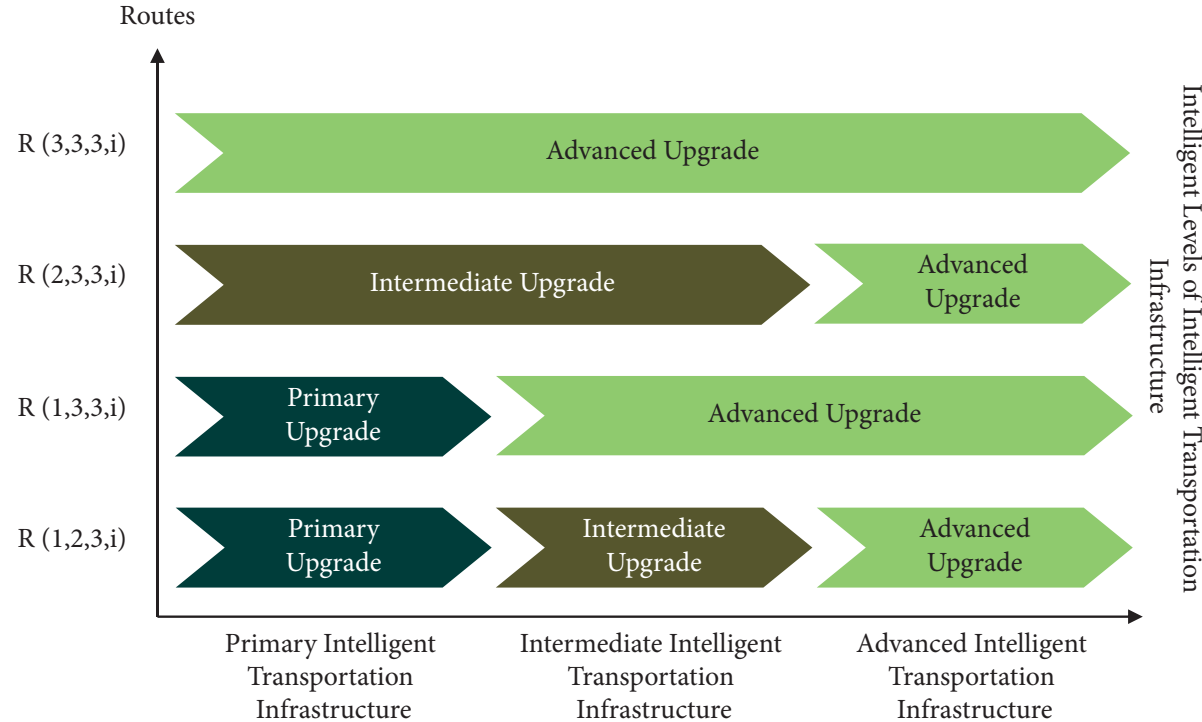

Figure 8: Intelligent upgrade routes of transportation infrastructure.

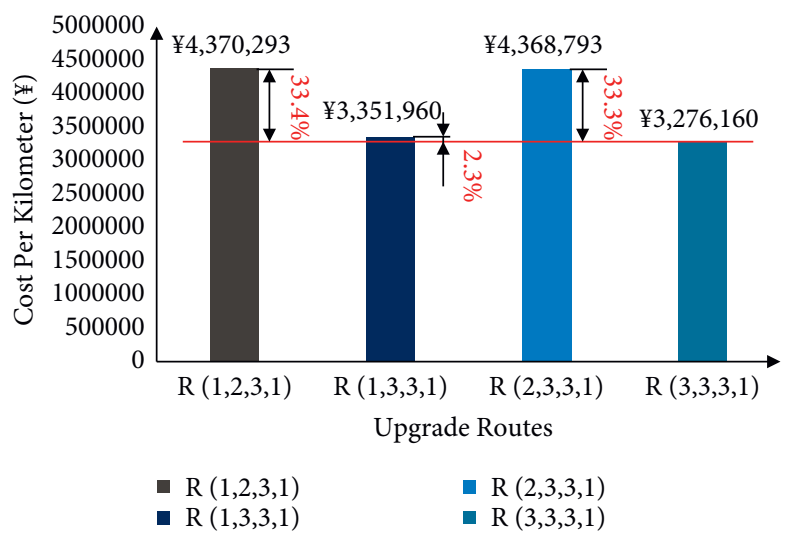

FIgURE 9: The total costs per kilometer of different upgrade routes on closed highways.

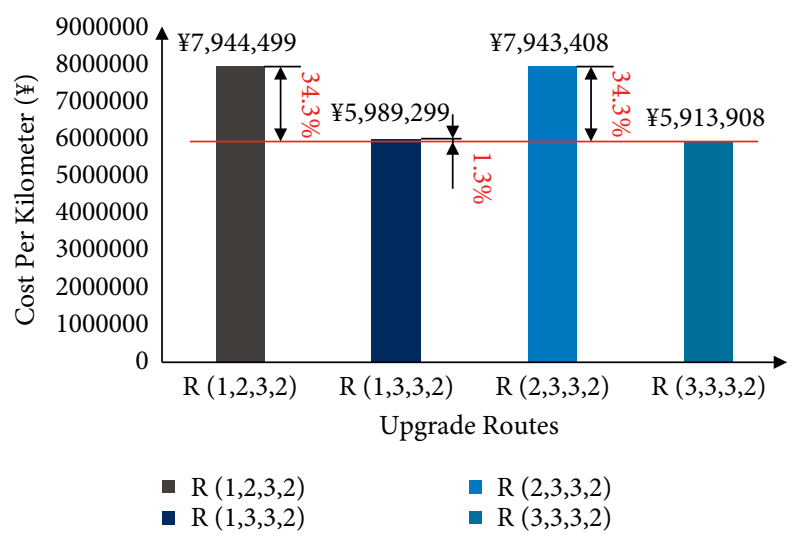

Figure 10: The total costs per kilometer of different upgrade routes on open urban roads. 


\subsection{Key Elements Affecting the Cost of Intelligent Upgrade of Transportation Infrastructure}

4.2.1. Identification of Key Elements. Figures 11 and 12 depict the share of cost components of one-time intelligent upgrade of transportation infrastructure in closed highway and open urban road scenarios. The cost components of the two scenarios are similar. In the primary stage, RSUs account for the highest cost ratio and far exceed other components due to their high costs and great demands. In the intermediate and advanced levels, LiDARs' costs are the highest, followed by RSUs' and edge computing servers', which are all due to their high costs. In addition, because the data transmission rate of $5 \mathrm{G}$ base stations has not yet reached the standard, the costs of $5 \mathrm{G}$ base stations in the advanced stage increase apparently compared to those in the intermediate stage. So, a large number of base stations have to be added to meet the demand for data transmission rate of every advanced ICV, which is approximately $100 \mathrm{Mbps}$. In summary, the data transmission rate of $5 \mathrm{G}$ base stations, as well as the costs of RSUs, LiDARs, and edge computing servers, are key factors affecting the total costs of intelligent transportation infrastructure.

4.2.2. Impact of Key Elements on the Upgrade Cost. Due to the lack of maturity and popularity of some new technologies, much transportation infrastructure is currently expensive. As the technologies gradually mature, the prices of related transportation infrastructure are expected to decline in the future [36]. Therefore, scenario analysis is adopted to analyze the sensitivity of the four key elements recognized above. There are four scenarios set for analysis:

(1) As for an RSU, it is assumed that the mature technology will reduce its cost by $50 \%$ to $¥ 35,000$.

(2) As for an edge computing server, it is assumed that the mature technology will reduce its cost by $50 \%$ to $¥ 87,500$.

(3) For LiDAR, it is assumed that the mature technology will reduce its cost by $50 \%$ of the primary and intermediate costs, reaching $¥ 101,834$.

(4) As for $5 G$ base stations, it is believed that the data transmission rate of $5 \mathrm{G}$ will eventually meet the standard, that is, be upgraded from $1 \mathrm{Gbps}$ to 10 Gbps.

The results of the scenario analysis of the impact of key elements on the upgrade cost for both scenarios are shown in Tables 5 and 6 . It is obvious that the total cost of intelligent upgrade of transportation infrastructure has changed significantly. $50 \%$ cost reduction of a LiDAR has the greatest impact on the costs of all four routes, saving $21.3 \%$ to $28.0 \%$ of the total cost on closed highways and $22.9 \%$ to $29.5 \%$ on open urban roads. $50 \%$ cost reduction of an RSU can save $8.0 \%$ to $10.7 \%$ of the total cost on closed highways and $5.4 \%$ to $7.3 \%$ on open urban roads. Similar to RSUs, $50 \%$ cost reduction of an edge computing server results in cost savings of $8.0 \%$ to $10.7 \%$ on closed highways and of $5.1 \%$ to $6.8 \%$ on open urban roads, respectively. The data transmission rate of

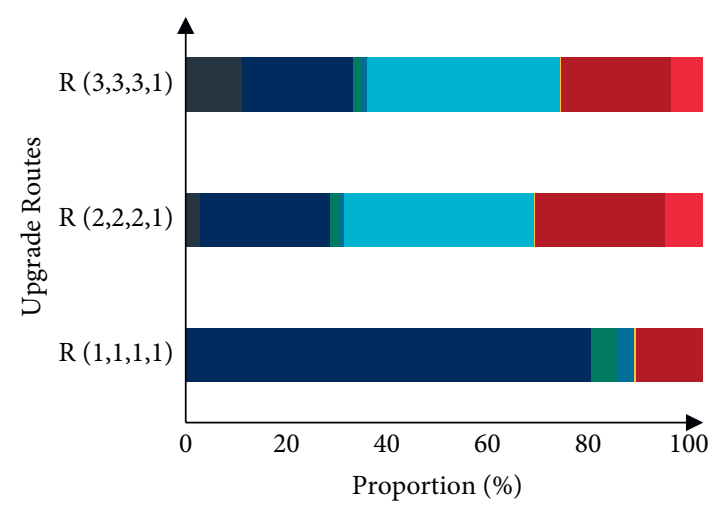

$$
\begin{aligned}
& \text { - LTE Base Stations } \\
& \text { - 5G Base Stations } \\
& \text { - RSUs } \\
& \text { - Vision Sensors } \\
& \text { - Millimete Wave Radars } \\
& \text { - LiDARS } \\
& \text { - Intelligent Signal Machines } \\
& \text { - Meteorological Sensors } \\
& \text { - Edge Computing Servers }
\end{aligned}
$$

FIGURE 11: Share of cost components of one-time intelligent upgrade of transportation infrastructure in closed highway scenario.

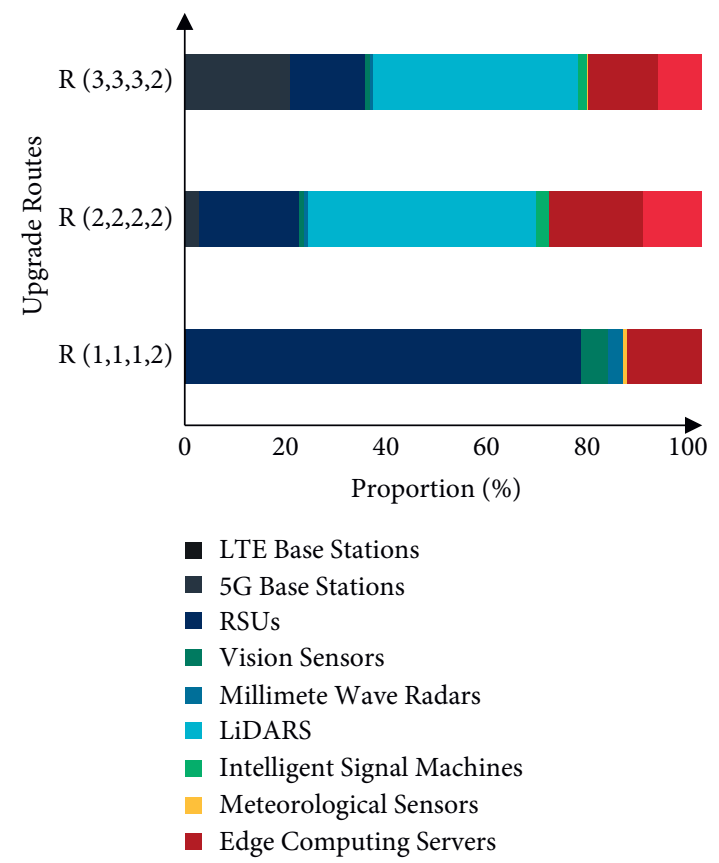

FIGURE 12: Share of cost components of one-time intelligent upgrade of transportation infrastructure in open urban road scenario.

standard-compliant $5 \mathrm{G}$ has a great impact on the upgrade cost on open urban roads, which can reduce cost by $13.6 \%$ to $18.3 \%$. This is due to the higher traffic volume on open urban roads, which requires a higher data transmission rate.

As a result, faced with the upgrade demand of advanced intelligent transportation infrastructure in the future, it is of great significance to promote the development of RSUs and edge computing servers and accelerate the technological 
TABLE 5: Scenario analysis: impact of key elements on the total upgrade cost per kilometer on closed highways.

\begin{tabular}{|c|c|c|c|c|}
\hline \multirow{2}{*}{ Scenario } & \multicolumn{4}{|c|}{ Route of closed highways } \\
\hline & $R(1,2,3,1)$ & $R(1,3,3,1)$ & $R(2,3,3,1)$ & $R(3,3,3,1)$ \\
\hline $50 \%$ cost reduction of an RSU & $-8.0 \%$ & $-10.4 \%$ & $-8.0 \%$ & $-10.7 \%$ \\
\hline $50 \%$ cost reduction of an edge computing server & $-8.0 \%$ & $-10.4 \%$ & $-8.0 \%$ & $-10.7 \%$ \\
\hline $50 \%$ cost reduction of a $\mathrm{LiDAR}$ & $-28.0 \%$ & $-21.3 \%$ & $-28.0 \%$ & $-21.8 \%$ \\
\hline The data transmission rate of standard-compliant $5 \mathrm{G}$ & $-6.5 \%$ & $-8.5 \%$ & $-6.5 \%$ & $-8.7 \%$ \\
\hline
\end{tabular}

TABLE 6: Scenario analysis: impact of key elements on the total upgrade cost per kilometer on open city roads.

\begin{tabular}{|c|c|c|c|c|}
\hline \multirow{2}{*}{ Scenario } & \multicolumn{4}{|c|}{ Route of open urban roads } \\
\hline & $R(1,2,3,2)$ & $R(1,3,3,2)$ & $R(2,3,3,2)$ & $R(3,3,3,2)$ \\
\hline $50 \%$ cost reduction of an RSU & $-5.4 \%$ & $-7.2 \%$ & $-5.4 \%$ & $-7.3 \%$ \\
\hline $50 \%$ cost reduction of an edge computing server & $-5.1 \%$ & $-6.7 \%$ & $-5.1 \%$ & $-6.8 \%$ \\
\hline $50 \%$ cost reduction of a LiDAR & $-29.5 \%$ & $-22.9 \%$ & $-29.5 \%$ & $-23.1 \%$ \\
\hline The data transmission rate of standard-compliant $5 \mathrm{G}$ & $-13.6 \%$ & $-18.0 \%$ & $-13.6 \%$ & $-18.3 \%$ \\
\hline
\end{tabular}

improvement and deployment process of $5 \mathrm{G}$, which is expected to effectively reduce the cost of intelligent upgrade of intelligent transportation infrastructure.

\section{Conclusion and Discussion}

In this study, intelligent levels and corresponding functions of transportation infrastructure were defined based on ICVs with different intelligent levels and relevant demands of ICV intelligent upgrade. Based on the existing intelligent transportation infrastructure products, the possible upgrade routes were analyzed, and the types and quantities of the required intelligent transportation infrastructure products were determined. Then the cost evaluation model of intelligent upgrade of transportation infrastructure was established, based on which the costs of four intelligent upgrade routes of transportation infrastructure were evaluated under closed highway and open urban way scenarios. Furthermore, combined with actual industry situation, the best route was recommended. At last, key elements that affect the upgrade costs were identified, and their impacts were evaluated through scenario analysis.

Several conclusions could be drawn from the study. First of all, on closed highways and open urban roads, the costs of completed intelligent upgrade of transportation infrastructure are $¥ 3,276,160$ to $¥ 4,370,293$ and $¥ 5,913,908$ to $¥ 7,944,499$, respectively. Next, considering the practical feasibility, the optimal route is to first build primary intelligent transportation infrastructure and then directly upgrade them to the advanced level, ignoring the intermediate level. Then, the costs of intelligent transportation infrastructure upgrade are less than one-seventh of the construction costs of ordinary road, which are not hard to afford, and it is possible to promote ICV development in V2X mode. At last, the costs of RSUs, edge computing servers, LiDARs, and data transmission rate of $5 \mathrm{G}$ transmission rate are four key elements exerting apparent impacts on the upgrade costs. Therefore, in the future, relevant technology maturation should be accelerated to reduce the costs of intelligent transportation infrastructure upgrades.

According to the evaluation results, it is feasible to accelerate the development of ICVs based on V2X mode so as to sufficiently obtain multiple benefits such as traffic efficiency, driving safety, energy conservation, and environmental protection. So, automobile, transportation, and information industries should pay more attention to $\mathrm{V} 2 \mathrm{X}$, especially the construction of future transportation systems, and match the intelligent functional requirements of ICVs on their own. In fact, the future human society is expected to present a new situation in which ICVs, smart transportation, and smart city develop and work in a coordinated and integrated way. As the only flexible mobile tool, vehicles are much likely to become the link and key terminal of smart transportation and smart city with the support of the Internet of Things. Hence, intelligent upgrades of transportation infrastructure based on ICV demands can generate important value to improve the overall operational efficiency and governance of a city and are expected to share the cost of V2X construction.

There are still some points to be optimized in this study. On the one hand, the cost evaluation model of intelligent upgrades of transportation infrastructure did not consider the costs of software algorithms, device operation and maintenance, manual labor, device transportation, and so on. On the other hand, only two scenarios were considered in this study, and it is necessary to analyze them in detailed road classification according to Chinese standards so as to get more comprehensive and accurate results. In addition, limited by the reliability and availability of data, the new devices which are still in the conceptual or research stage were not considered. As a matter of fact, the current pace of technological progress in the field of ICVs and smart transportation is very fast, so it is necessary to carry out the research on a rolling basis to make conclusions fit the latest situation of the industries at any time. 


\section{Data Availability}

The data used to support the findings of this study were collected from research reports and appliers, which are available from the corresponding author.

\section{Conflicts of Interest}

The authors declare that they have no conflicts of interest.

\section{Acknowledgments}

This research was supported by the National Natural Science Foundation of China (U1764265).

\section{References}

[1] Z. W. Liu, Zhao Fuquan's Insights on the Automotive Industry, China Machine Press, Beijing, China, 2020.

[2] K. Q. Li, Annual Report on the Development of China's Intelligent Connected Vehicle Industry, Social Sciences Academic Press, Beijing, China, 2019.

[3] B. van Arem, C. J. G. van Driel, and R. Visser, "The impact of cooperative adaptive cruise control on traffic-flow characteristics," IEEE Transactions on Intelligent Transportation Systems, vol. 7, no. 4, pp. 429-436, 2006.

[4] C. Stogios, D. Kasraian, M. J. Roorda, and M. Hatzopoulou, "Simulating impacts of automated driving behavior and traffic conditions on vehicle emissions," Transportation Research Part D: Transport and Environment, vol. 76, pp. 176-192, 2019.

[5] Y. Lu, X. Xu, C. Ding, and G. Lu, "A speed control method at successive signalized intersections under connected vehicles environment," IEEE Intelligent Transportation Systems Magazine, vol. 11, no. 3, pp. 117-128, 2019.

[6] A. Olia, S. Razavi, B. Abdulhai, and H. Abdelgawad, "Traffic capacity implications of automated vehicles mixed with regular vehicles," Journal of Intelligent Transportation Systems, vol. 22, no. 3, pp. 244-262, 2018.

[7] D. Milakis, B. van Arem, and B. van Wee, "Policy and society related implications of automated driving: a review of literature and directions for future research," Journal of Intelligent Transportation Systems, vol. 21, no. 4, pp. 324-348, 2017.

[8] A. Olia, H. Abdelgawad, B. Abdulhai, and S. N. Razavi, "Assessing the potential impacts of connected vehicles: mobility, environmental, and safety perspectives," Journal of Intelligent Transportation Systems, vol. 20, no. 3, pp. 229-243, 2016.

[9] L. Qiu, L. Qian, Z. Abdollahi, Z. Kong, and P. Pisu, "Enginemap-based predictive fuel-efficient control strategies for a group of connected vehicles," Automotive Innovation, vol. 1, no. 4, pp. 311-319, 2018.

[10] T. Kuehbeck, G. Hakobyan, A. Sikora, C. C. Chibelushi, and M. Moniri, "Evaluation of performance enhancement for crash constellation prediction via car-to-car communication," in Proceedings of the International Workshop on Communication Technologies for Vehicles, pp. 57-68, Midtown Manhattan, NY, USA, 2014.

[11] H. Tan, F. Zhao, H. Hao, and Z. Liu, "Estimate of safety impact of lane keeping assistant system on fatalities and injuries reduction for China: scenarios through 2030," Traffic Injury Prevention, vol. 21, no. 2, pp. 156-162, 2020.

[12] Y. Li, Z. Li, H. Wang, W. Wang, and L. Xing, "Evaluating the safety impact of adaptive cruise control in traffic oscillations on freeways," Accident Analysis \& Prevention, vol. 104, pp. 137-145, 2017.

[13] F. Q. Zhao, Z. W. Liu, and K. Q. Yang, Automotive Technology Innovation, China Machine Press, Beijing, China, 2019.

[14] K. Q. Li, Y. F. Dai, and S. B. Li, "State-of-the-Art and technical trends of intelligent and connected vehicles," Journal of Automotive Safety and Energy, vol. 8, no. 1, pp. 1-14, 2017.

[15] Y. P. Wang and X. P. Yan, Introduction to Intelligent Transportation Technology, Tsinghua University Press, Beijing, China., 2020.

[16] X. Y. Chang, Intelligent and Connected Vehicles' Cloud Control System and its Control Technology, Tsinghua University (Doctor's thesis), Beijing, China, 2020.

[17] H. Wieker, J. Vogt, and M. Fuenfrocken, "Intelligent transportation system infrastructure and software challenges," in Automotive Systems and Software Engineering, pp. 295-319, Springer, Cham, Midtown Manhattan, New York City, 2019.

[18] J. M. León-Coca, D. G. Reina, S. L. Toral, and F. Barrero, "Intelligent transportation systems and wireless access in vehicular environment technology for developing smart cities," Big Data and Internet of Things: A Roadmap for Smart Environments, pp. 285-313, Springer, Cham, Midtown Manhattan, NY, USA, 2014.

[19] M. Mirboland and K. Smarsly, "BIM-based description of intelligent transportation systems for roads," Infrastructure, vol. 6, no. 4, p. 51, 2021.

[20] J. Xu, L. Wu, L. Shi, Y. Shi, and W. Zhou, "Research on 5G Internet of vehicles facilities based on coherent beamforming," in Proceedings of the International Conference on Wireless Algorithms, Systems, and Applications, pp. 68-77, Midtown Manhattan, NY, USA, 2020.

[21] X. Zhang, J. Jiang, L. Qi, and X. Xi, “An Edge Server Placement Method with Cyber-Physical-Social Systems in 5G," in Proceedings of the 12th EAI International Conference in Simulation Tools and Techniques SIMUtools 2020, August 28-29, 2020, Proceedings, Part II 12, pp. 127-139, Guiyang, China, August 2020.

[22] W. Zhang, Research on Layout Optimization of Hybrid VANET-Sensor Network for Autonomous Driving, Master's thesis, Dalian Maritime University, Dalian, China, 2019.

[23] H. Zhu, Research of RSU Deployment Algorithms for VANET, Master's thesis, Dalian Maritime University, Dalian, China, 2016.

[24] F. G. Abdulkadhim, Z. Yi, A. N. Onaizah, F. Rabee, and A. M. A. Al-Muqarm, "Optimizing the roadside unit deployment mechanism in VANET with efficient protocol to prevent data loss," Wireless Personal Communications, pp. 1-29, 2021.

[25] M. Al Shareeda, A. Khalil, and W. Fahs, "Towards the Optimization of Roadside Unit Placement Using Genetic Algorithm," in Proceedings of the 2018 International Arab Conference on Information Technology (ACIT), pp. 1-5, Islamic University of Lebanon, Lebanon, November 2018.

[26] L. Sun, Y. Wu, J. Xu, and Y. Xu, "An RSU-Assisted Localization Method in Non-GPS Highway Traffic with Dead Reckoning and V2R Communications," in Proceedings of the 2012 2nd International Conference on Consumer Electronics, Communications and Networks (CECNet), pp. 149-152, Yichang, China, April 2012.

[27] H. J. Li, H. H. Dong, D. W. Xu, and L. Jia, "Urban road traffic oriented sensor networking optimization," Journal of Central South University, vol. 43, no. 3, pp. 1192-1197, 2012.

[28] X. Xu, H. K. Lo, A. Chen, and E. Castillo, "Robust network sensor location for complete link flow observability under 
uncertainty," Transportation Research Part B: Methodological, vol. 88, pp. 1-20, 2016.

[29] F. Zhan, X. Wan, Y. Cheng, and B. Ran, "Methods for multitype sensor allocations along a freeway corridor," IEEE Intelligent Transportation Systems Magazine, vol. 10, no. 2, pp. 134-149, 2018.

[30] A. Danczyk, X. Di, and H. X. Liu, "A probabilistic optimization model for allocating freeway sensors," Transportation Research Part C: Emerging Technologies, vol. 67, pp. 378-398, 2016.

[31] Strategic Advisory Committee of Energy-Saving and New Energy Vehicle Technology Roadmap, "China Society of Automotive Engineering," Energy-saving and New Energy Vehicle Technology Roadmap 2.0, China Machine Press, Beijing, China., 2020.

[32] China Association for Road Traffic Safety, Roadside Facilities Setting Guide for Internet of Vehicles, Standards Press of China, Beijing, China, 2020.

[33] Sinolink Securities, Research on Communication Industry, Sinolink Securities, Shanghai, China, 2020.

[34] Z. Y. Yang, W. S. Liu, and S. Zhang, V2X Internet of Vehicles: The Leader of $5 G$ New Infrastructure, Citic Securities, Beijing, China, 2020.

[35] X. Kuang, Research on the Benefits and Business Models of Intelligent Connected Vehicles, Doctor's thesis, Tsinghua University, Beijing, China, 2019.

[36] M. A. Schilling, W. Xie, Y. Wang, and P. X. Li, Strategic Management of Technology Innovation, Tsinghua University Press, Beijing, China, 2005. 